\author{
Mass Spectrometry PittCon '95 \\ Reviewed by O. David Sparkman \\ Mass Spectrometry Consultant \\ 4045 East Ridge Drive \\ Antioch, CA 94509
}

This review is for the benefit of our readers. Its content is the responsibility of the reviewer and not of the ASMS.

Mass spectrometry was a dominant part of the 1995 Pittsburgh Conference held in New Orleans, Louisiana, this March 5th to the 10th. In addition to one of the highest quality series of technical presentations on mass spectrometry at a PittCon, there were nine separate short courses on different areas of mass spectrometry offered with over 200 people in attendance. All the major manufacturers presented products that were new or had enhanced features on already marketed products. In the area of organic mass spectrometry, there were at least five companies that offered new technology instruments. The only negative note was the absence of the ATI-Unicam Automass (formerly the Nermag Automass) transmission quadrupole GC/MS. Informed sources in the ATI organization said the instrument is no longer being offered in the United States.

The new technology introductions were somewhat eclipsed by the news that Thermo Instruments Systems was acquiring the Scientific Instruments Division of Fisons. Since Shimadzu discontinued the magnetic sector instrument activities of Kratos, only Thermo (the Finnigan group and the Fisons group) and JEOL are manufacturers of sector-based instruments for organic analyses now. Reliable sources within Perkin-Elmer Sciex said they were looking into the antitrust and international cartel implications of this acquisition. A number of users voiced concerns as to how this merger would affect future developments in instrumentation. Dr. Richard W. K. Chapman, President of Finnigan Corporation, said the current mass spectrometry product line of Thermo would be complimented by the acquisition of Fisons rather than replicated. Thermo is now the largest mass spectrometry company and the second largest seller of analytical instruments, just behind Perkin-Elmer, which had sales of $\$ 1.02$ billion for the fiscal year ending June 30, 1994.

\section{Finnigan MAT}

The most promoted new product offered in mass spectrometry was the Finnigan MAT GCQ. This is an ion trap mass spectrometer that uses the electron ionization (EI) source (convertible to a chemical ionization (CI) source by changing the ion volume) of Finnigan's research grade quadrupole mass spectrometers. Ions are formed outside the ion trap and then stored in the trap. The instrument is capable of both positive and negative ion detection and has a mass range of 1000 $\mathrm{Da}$. The instrument will allow for electron capture negative ion detection with a sensitivity specification of $1 \mathrm{pg}$ hexachlorobenzene producing a chromatographic peak for $m / z 284$ with à signal-to-noise ratio of at least 100:1 when the instrument is scanned 50 to $300 \mathrm{Da}$ at a rate of two scans/s. The base selling price of the instrument with EI/CI source, positive/negative ion detection, and selected ion monitoring (SIM) is $\$ 63,000$. This includes a specially designed Tremetrics GC with electronic pressure control (EPC) and a very powerful data system with newly designed Windows

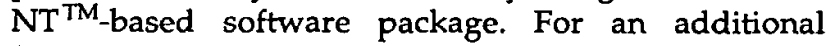
$\$ 5,000$, you can add MS $/ M S$. There are a number of other options, such as a vacuum interlock for quick change of ion volumes and the use of direct insertion and desorption chemical ionization probes, an ion gauge, and a jet separator or open-split interface. The instrument is also available with an $\mathrm{HP}$, Varian, or 9001 Tremetrics GC. In addition to the external ion source on the GCQ, its vacuum system is a $180 \mathrm{~L} / \mathrm{sec}$ diffusion pump with a Peltier cooled baffle. Most all other ion trap products on the market today use turbomolecular pumps. A source of helium is supplied to the ion trap separate from the GC column. This way, the buffer gas partial pressure will not vary with changing flow rates from the column.

At the Finnigan press conference, Dr. Ian Jardine, Director of Marketing, also talked about another new product from Finnigan MAT, the Element ICP/GD Mass Spectrometer. This is a high resolution ICP-MS that is of particular interest to the semiconductor industry. This instrument allows for the separation of ${ }^{56} \mathrm{Fe}$ and ${ }^{40} \mathrm{Ar}{ }^{16} \mathrm{O}$.

\section{Meridian Analytical Systems}

Meridian Analytical Systems of Okemos, MI, introduced a GC/MS based on a time-of-flight mass spectrometer. The Fast Chromatographic Detector (FCD) can be interfaced to a Fast GC or a conventional GC. Using their patented integrating transient recorder (ITR), the developers demonstrated a data acquisition rate of $100 \mathrm{spectra} / \mathrm{s}$ over a mass range of $650 \mathrm{Da}$ (remember, a TOF MS does not scan). According to Dr. Peter Burrill, Vice President, Meridian has sold an instrument that will allow for an acquisition rate of a 1000 spectra/s over a mass range of $1000 \mathrm{Da}$ with a flow of $5 \mathrm{~mL} / \mathrm{min}$ from the $\mathrm{GC}$.

The FCD allows for a flow rate of $2 \mathrm{~mL} / \mathrm{min}$ and has a sensitivity in the low picogram range. The most outstanding feature is the ability to do time-compressed chromatography (TCC). This is another Merid- 
ian patented process by which the speed of the analysis is enhanced by purposefully reducing the chromatographic resolution and then separating the components by use of the mass spectral data. Because there is minimal spectral skewing in the TOF MS data, it is possible to resolve all the components. A GC/MS analysis of 55 volatile organic components, where all the components were identified and quantitated, was accomplished in 14 seconds. This technology, coupled with Varian's automated solid phase micro extraction (SPME) and Fast GC (both offered by Meridian), may be the future for GC/MS.

The current selling price of the entry level FCD is $\$ 125,000$. This includes a data system running on a high performance 486 PC using the UNIX operating system but does not include the gas chromatography. Meridian offers both the HP and Varian GC.

\section{Bear Instruments}

Another new product in organic mass spectrometry was the Bear Instruments, Inc. CUB 800 triple quadrupole mass spectrometer. This benchtop instrument, not much larger than a bread box, has several unique features. The instrument uses three hyperbolic quadrupoles that are arranged end-to-end in a circular fashion. The quadrupoles are held together with a unique mounting feature that allows for a wireless construction. This allows for the entire instrument to be housed in an enclosure that is $18^{\prime \prime}$ wide, $21^{\prime \prime}$ deep, and $14.5^{\prime \prime}$ high. The current instrument has a mass range of $800 \mathrm{Da}$; and, according to company founder Urs Steiner, it can be expanded to $3000 \mathrm{Da}$ with a resolving power of $0.1 \mathrm{u}$ and a scan rate of 10,000 $\mathrm{Da} / \mathrm{s}$. The instrument uses a single $250 \mathrm{~L} / \mathrm{sec}$ turbomolecular pump that is in the same housing as the mass spectrometer. The pump is multiported to allow for differential pumping of three separate compartments. The instrument has a complete mass spectrometry data system based on a $90 \mathrm{MHz}$ Pentium computer and using Windows $\mathrm{NT}^{\mathrm{TM}}$. There are no inlets currently available, and the company is looking for a partner to help in the development of inlets and markets. The current manufacturing cost requires a selling price of about $\$ 100,000$, but Mr. Steiner reportedly said that it could be produced in quantity at a cost that would allow for a selling price of $\$ 50,000$ before inlet systems are added.

\section{PE Sciex}

Another new product introduction that will have a significant impact on the industry is the API 100 and API 300 from Perkin-Elmer Sciex, which are now sold by the Applied Biosystems Division of Perkin-Elmer. The API 100 is a single quadrupole mass spectrometer using machined ceramic rods plated with titanium, nickel, and gold. The instrument has a mass range to $3000 \mathrm{Da}$ and a price of $\$ 150,000$ with an IonSpray ${ }^{\mathrm{TM}}$ interface. The API 300 is the triple-quad version of the API 100. Both instruments are very small and can be mounted on your benchtop or a special optional table available from PE Sciex. Both are supplied with a 110 $\mathrm{MHz}$ Power Macintosh ${ }^{8}$ RISC data system that uses proprietary Macintosh software for multitasking instrument control and data analysis under a graphical interface.

There are three different IonSpray ${ }^{\mathrm{TM}}$ interfaces that offer flow rates of 100 to $5000 \mathrm{~nL} / \mathrm{min}, 1$ to 200 $\mu \mathrm{L} / \mathrm{min}$ (up to $10 \mathrm{~mL} / \mathrm{min}$ with splitting) and up to 2 $\mathrm{mL} / \mathrm{min}$ without splitting. In addition, there is a heater nebulizer atmospheric pressure chemical ionization source. All of these different interfaces are interchangeable between the two instruments and clamp on the analyzer with easy on/off connectors.

\section{JEOL}

This was truly the year of the smaller-is-better benchtop mass spectrometer. JEOL introduced the GCMate Benchtop High Resolution GC/MS. This instrument was first shown to the western world at the 1994 International Mass Spectrometry Conference in Budapest. It has a mass range of 1 to $1000 \mathrm{Da}$ and a maximum resolving power of 5000 . It has a scan rate of 0.07 seconds per decade, has a combination $\mathrm{EI} / \mathrm{CI}$ ion source, differential pumping using turbomolecular pumps, and a data system developed on a PC using Windows $^{\mathrm{TM}}$ by Steve Shrader of Shrader Analytical Labs in Detroit, MI. According to Dr. Brian Musselman, Product Manager of JEOL USA, Inc., the selling price of this double-focusing GC/MS with an HP GC is $\$ 170,000$. The mass spectrometer portion of the instrument has a footprint of $27^{\prime \prime}$ wide by $34^{\prime \prime}$ deep. An LC/MS interface and positive and negative ion detection are available.

\section{Bruker Instruments}

Bruker has been providing a variety of instruments for biomass spectrometry for a number of years. Last year, they introduced a field transportable GC/MS based on a transmission quadrupole mass spectrometer. This year, they introduced the same instrument using the quadrupole ion trap. The instrument uses an ion getter pump to reduce power consuption and instrument weight. Both the portable instruments use an OS/2 based data system.

Bruker also offers quadrupole ion trap mass spectrometers with external electrospray ionization, MALDI-TOF instruments, and FT ion cyclotron resonance mass spectrometers. The BioAPEX ${ }^{\mathrm{TM}}$, an ion cyclotron resonance mass spectrometer, was shown this year with shielded magnet which allows for a smaller instrument and no-stray magnetic fields. This instrument, with a resolving power of greater than $100,000 \mathrm{Da}$, uses a Silicon Graphics based work station. 


\section{Teledyne Electronic Technologies}

Teledyne introduced the 3DQ Apogee ${ }^{\mathrm{TM}}$, "an advanced feature upgrade for older, outdated ion traps," according to their press release. This product is directed toward Varian Saturn and Finnigan MAT ITS40/Magnum users. At $\$ 30,000$ installed, it adds a number of new features to existing ion trap GC/MS. It allows for use of Teledyne's patented technology for selected ion monitoring and MS/MS in the ion trap. The kit replaces some electronic boards in the mass spectrometer and adds a true Windows ${ }^{\mathrm{TM}}$-based data system. The price also includes a new computer and monitor. Teledyne also showed its 3DQ Discovery ion trap mass spectrometer, introduced at PittCon 94.

\section{Too Many to Include All Individually}

While many of the other companies did not show new products in mass spectrometry this year, they were there with some of the highest quality instrumentation ever available. The Fison's Organic MS group has a Windows ${ }^{\mathrm{TM}}$-based data system that crosses the lines of many of its products. Varian showed the Satum ion trap GC/MS with its 4D MS/MS and selected ion storage options. Hewlett-Packard showed the 5972 with its newly introduced $6890 \mathrm{GC}$, the very popular and practical GCD, and their benchtop ICP-MS. Both the GCD and the benchtop ICP-MS brought a new dimension to analytical mass spectrometry at last year's PittCon. Shimadzu showed the QP 5000 transmission quadrupole GC/MS with its Windows ${ }^{\mathrm{TM}}$-based data system. Perkin-Elmer added some features to the data system of the Q-Mass, a quadrupole GC/MS system. They introduced Version 4.5 of the NIST Mass Spectral Search Program and new environmental reporting software based on a port of the HP Aquarius 2000 soft- ware to a PC. The Q-Mass now uses a new Prevent injector that PE introduced on its GC line. Hitachi Instruments showed its version of the Teledyne ion trap mass spectrometer with an HP GC. PerSeptive Biosystems introduced the Voyager ${ }^{\mathrm{TM}}$-Elite Biospectrometry $^{\mathrm{TM}}$ Workstation for its MALDI-TOF/MS, and Waters showed their FT-ICR MS and Integrity transmission quadrupole LC/MS with a particle beam interface.

In addition to all these hardware advances in organic mass spectrometry, there was a lot of other activity in the areas of ICP-MS and secondary ion mass spectrometry. Several third-party hardware support organizations and mass spectrometry resellers exhibited as well. LGC of Los Gatos, CA showed a new Windows ${ }^{\mathrm{TM}}$-based data system, the Galaxy 2000 , for use with transmission quadrupole and ion trap instruments. It offers both automated data reduction and instrument control. Mass Evolution of Houston, TX, introduced EZScan ${ }^{\oplus}$, a GC/MS and GC/MS/MS data system for use with quadrupole mass spectrometers. The data reduction software is the Windows ${ }^{\mathrm{TM}}$-based program from Shrader Analytical Labs. NIST showed its new Windows ${ }^{\mathrm{TM}}$-based program for use in searching EI mass spectra in its database of 62,235 compounds and the identification of unknown compounds. Palisade Corp. of Newfield, NY, showed its Windows ${ }^{\mathrm{TM}}$-based mass spectral search program and MASSTransit ${ }^{\mathrm{TM}}$, a program used to convert most any MS or MS library data file format to most any other format (a must for people with multiple data systems from different manufacturers). New programs to aid in the evaluation of EI mass spectra were presented by WindowsChem (Fairfield, CA) and SoftShell (Grand Junction, CO). SAVANT of Fullerton, CA showed MS Fundamentals, a multimedia training program for GC/MS. 